\title{
Numerical Solution of Some Classical Differential-Difference Equations*
}

\author{
By George Marsaglia, Arif Zaman, and John C. W. Marsaglia
}

\begin{abstract}
For differential-difference equations, we provide a method that gives numerical solutions accurate to hundreds or even thousands of digits. We illustrate with numerical solutions to three classical problems. With a few exceptions, previous claims of extended accuracy for these problems are found to be wrong.
\end{abstract}

1. Introduction. This article describes a method for evaluating functions defined by differential-difference equations. It concentrates on three specific functions that, because they have been discussed in scores of papers going back over fifty years, may be considered classical. Subject to various initial conditions, with a prime indicating differentiation, the defining relations for the three functions are:

$$
\begin{aligned}
& \text { Renyi's function: } \quad[(x-1) f(x)]^{\prime}=2 f(x-1) \\
& \text { Dickman's function: } \quad x v^{\prime}(x)=-v(x-1) \\
& \text { Buchstab's function: } \quad[x w(x)]^{\prime}=w(x-1)
\end{aligned}
$$

We will describe a method that provides simple and very accurate evaluation of these functions, given their forms over an initial interval. The method then builds up the function over successive intervals - as do all the published methods, but rather than use previous methods, involving numerical integration, we suggest a series method that seems simpler and is much more accurate.

2. Previous Results. Numerous authors have reported numerical values for solutions of the above equations. As far as can be determined, all were based on numerical integration. Values for Renyi's function were published by Manion [13] and by Blaisdell and Solomon [4]. Davenport and Erdős [5] gave a few values of Dickman's function, and extensive numerical integrations were carried out by Bellman and Kotkin [1], van de Lune and Wattel [12] and by Lal and Gillard [11], who also carried out extensive numerical integrations of Buchstab's function.

Except for van de Lune and Wattel, who exploited the convexity of Dickman's function and gave a masterly analysis of their numerical integration, other numerical solutions have been found to have accumulated error far in excess of their authors' claims. The Bellman and Kotkin [1] results are unacceptable beyond $x=9$, Blaisdell and Solomon's [4] are bad beyond $x=13$ or so, and the results claimed in Lal

Received May 13, 1988.

1980 Mathematics Subject Classification (1985 Revision). Primary 65D30, 65Q05; Secondary $39 \mathrm{~A} 10$. tions.

Key words and phrases. Difference equations, differential-difference equations, numerical solu-

*Research supported in part by the National Science Foundation, grant DMS-8807976 and the Department of Energy contract DE-FC05-85ER250000. 
and Gillard [10] are far from correct beyond about $x=25$. With our new method, we have been able to provide numerical results with hundreds of places of accuracy out to $x=500$, using a PC AT. We will comment on the extent and accuracy of previous results in sections devoted to each of the three differential-difference equations.

The frequency and the magnitude of errors in published solutions to these differential-difference equations raises serious doubts about the suitability of the numerical integration methods used-usually Romberg or some other application of Richardson extrapolation. Error analysis based on the magnitude of high-order derivatives may not be suitable for equations whose solutions are functions that are generalized splines, having only a limited number of derivatives at the knots.

3. Ideas Behind the New Method. Differential-difference equations may be solved by converting them to equivalent integral-difference equations. For example, Renyi's function may be described by the conditions:

$$
f(x)= \begin{cases}2 & \text { for } 1 \leq x \leq 2 \\ \frac{2}{x-1} \int_{0}^{x-1} f(y) d y & \text { for } 2<x\end{cases}
$$

The solution is a generalized spline function with knots at $1,2,3, \ldots$ It is continuous at $x=1$. It has a continuous first, but no second, derivative at $x=2$; it has a continuous second, but no third, derivative at $x=3$, and so on. Between knots, the function $f$ is analytic, expressible as a power series, infinitely differentiable. The coefficients of successive power series change over each interval, but the defining conditions for $f$ allow the coefficients for a given interval to be simply expressed in terms of those for the previous interval.

The problem is to choose the form of each power series. If the expansion is about the left end point of the interval, convergence is too slow at the right end point. But expansions about the midpoint of each interval provide excellent convergence - equal to that arising from Chebyshev expansions (our first approach), and much simpler. We now give details, with a section for each of the three classical differential-difference equations converted to an integral-difference equation.

4. Renyi's Function. Here we have

$$
f(x)= \begin{cases}2 & \text { for } 1 \leq x \leq 2 \\ \frac{2}{x-1} \int_{0}^{x-1} f(y) d y & \text { for } 2<x\end{cases}
$$

This function arises from a random "parking" problem, posed by Schmetterer and solved by Renyi [15]. Let $f(x)=M(x)+1$ with $M(x)$ the mean number of random unit intervals, "cars", placed without overlap on a "street" of length $x$. The first car occupies the interval $(u, u+1)$, with $u$ chosen uniformly from 0 to $x-1$. This leaves two new, shorter, "streets" which may be independently covered with random nonoverlapping cars, and the process continues until only substreets of lengths less than 1 remain.

With $M(x)$ the expected number of cars placed, a divide-and-conquer approach allows one to write

$$
M(x)=1+\int_{0}^{x-1} \frac{M(u)+M(x-u-1)}{x-1} d u=1+\frac{2}{x-1} \int_{0}^{x-1} M(u) d u
$$


and thus, with $f(x)=M(x)+1$,

$$
f(x+1)=\frac{2}{x} \int_{0}^{x} f(y) d y .
$$

For $1 \leq x \leq 2$, only one car may be parked, so that $M(x)=1$ and $f(x)=2$ for $1 \leq x \leq 2$. (Alternative initial conditions arise from putting $M(x)=0$ for $0 \leq x \leq 1$, streets which can take no cars, leading to $M(x)=1$ and $f(x)=2$ for $1 \leq x \leq 2$.)

Using the Laplace transform of $M(x)$, Renyi showed that $M(x)=c x+c-1+$ $O\left(x^{-m}\right)$, or in current notation, $f(x)=c x+c+O\left(x^{-m}\right)$, with

$$
c=\int_{0}^{\infty} \exp \left(-2 \int_{0}^{t} \frac{1-e^{-u}}{u} d u\right) d t
$$

Subsequently, Dvoretsky and Robbins [7] gave more precise asymptotic behavior:

$$
f(x)=c x+c+O\left(\left(\frac{2 e}{x}\right)^{x-3 / 2}\right) .
$$

Evaluation of the constant $c$, which gives the asymptotic density of random packing on the line, has been the object of numerous research efforts. It has been conjectured that the asymptotic density of the random packing of unit squares in a big square is $c^{2}, c^{3}$ for unit cubes in a big cube, and so on; see [4]. One of us (G. Marsaglia, in 1971) computed $c$ to 31 places, using an early version of the method of this article, with Chebyshev series.

In a 1970 paper, Blaisdell and Solomon [4] reported that the best estimate of $c$ to that time was that of Manion [13]: $c=.74759$. They integrated Renyi's equation numerically to $x=15$ and made the explicit claim:

$$
.7475979202533978 \leq c \leq .7475979202533981 \text { (?) }
$$

The last four digits are wrong; rather than between 3978 and 3981 they should be 4114.

Lal and Gillard [10] computed $c$ to 19 places (correctly), using numerical integration of the integral-difference equation. They concluded with a note added in proof, claiming recent computations of Lal and Tiller gave $c$ to 44 places:

$$
c=.74759792025341143517873094363652421026172439 \text { (?) }
$$

That representation of $c$ is only accurate to 27 places; we have indicated the incorrect digits in bold face.

In fact, the method described here shows the value of $c$ to 100 places to be:

$$
\begin{aligned}
c= & .74759792025341143517873094383017817302478626407422 \\
& 83766042291634251678816029544043124308503693141112 \text { (!) }
\end{aligned}
$$

and, should anyone want it, we have $c$ to 1000 places. Of course there is little value in such great precision in $c$ itself, but there is considerable value in being able to provide great precision in early and intermediate stages of the recursive calculations necessary to solve differential- or integral-difference equations of the type discussed here. 
To provide an independent evaluation and check of the 100 digits in $c$ above, we have calculated $c$ directly by Renyi's integral representation. Details are in the Addendum.

We now turn to details of our method for evaluating Renyi's function $f$. If, for given integer $n, f(n+t)$ is known for $0 \leq t \leq 1$, then $f(n+1+t)$ may be determined from this version of relation (1):

$$
f(n+1+t)=\frac{n f(n+1)+2 \int_{0}^{t} f(n+y) d y}{n+t}, \quad 0 \leq t \leq 1 .
$$

In particular, if $f(n+t)$ is a known power series in $t$ that converges for $0 \leq t \leq 1$ then $f(n+1+t)$ will have a corresponding representation as a power series in $t$, with convergence for $0 \leq t \leq 1$. The coefficients of the new power series may be determined by integrating the series representation of $f(n+y)$ in the right side of relation (2).

This is probably the simplest way to build up representations of $f(x)$ over successive intervals: 1 to 2,2 to 3,3 to 4 , etc., but that method requires evaluation of power series in $t$ at $t=1$, with slow convergence. A more accurate method assumes power series about the midpoints of the intervals, leading to simple and very accurate evaluation of functions defined by relations such as the one in (1).

Accordingly, assume that, for given $n$, we have a representation

$$
f\left(n+\frac{1}{2}+\frac{1}{2} z\right)=a_{0}+a_{1} z+a_{2} z^{2}+a_{3} z^{3}+\cdots
$$

that converges for $-1 \leq z \leq 1$, and we seek a corresponding series

$$
f\left(n+1+\frac{1}{2}+\frac{1}{2} z\right)=b_{0}+b_{1} z+b_{2} z^{2}+b_{3} z^{3}+\cdots .
$$

Relation (2) may be put in the form

(5) $\left(n+\frac{1}{2}+\frac{1}{2} z\right) f\left(n+1+\frac{1}{2}+\frac{1}{2} z\right)=n f(n+1)+\int_{-1}^{z} f\left(n+\frac{1}{2}+\frac{1}{2} v\right) d v$.

Then the coefficients $b_{0}, b_{1}, \ldots$ are related to $a_{0}, a_{1}, \ldots$ by

$$
\begin{aligned}
& (2 n+1+z)\left(b_{0}+b_{1} z+b_{2} z^{2}+\ldots\right) \\
& \quad=2 n f(n+1)+2\left[a_{0} v+a_{1} v^{2} / 2+a_{2} v^{3} / 3+\cdots\right]_{v=-1}^{v=z}
\end{aligned}
$$

Thus, since $f(n+1)=a_{0}+a_{1}+a_{2}+\cdots$,

$$
b_{0}=\frac{2}{2 n+1}\left[(n+1) a_{0}+(n-1 / 2) a_{1}+(n+1 / 3) a_{2}+\cdots\right]
$$

and

$$
b_{i}=\left[2 a_{i-1} / i-b_{i-1}\right] /(2 n+1) \text { for } i=1,2,3, \ldots .
$$

The resulting series (4) provides extended precision evaluation of $f(x)$ over the interval in question. The series is alternating (after the first few terms), and its tail is bounded by a geometric series. Thus with an extended precision package - such as one we have written for a PC AT, with precision limited only by memory capacity, typically providing the binary equivalent of over a thousand decimal digits - we 
are able to evaluate $f(x)$ with great accuracy. Such accuracy requires that the $b_{0}, b_{1}, b_{2}, \ldots$ be at least as accurate, of course, but that is readily accomplished: $b_{1}, b_{2}, \ldots$ are all exact, rational multiples of $b_{0}$, which is itself the sum of a wellbehaved series. If we evaluate $b_{0}$ to, say, 1000 places, then we may evaluate any $f(x)$ in the interval in question to similar accuracy.

We have used recursive developments of the successive power series to get representations of $f$ for intervals $n \leq x \leq n+1$ out to $x=500$. We are thus able to get not only particular values of $f(x)$ but the shape of the entire function over that range. The function $f(x)$, shown in Figure 1a, oscillates around $c x+c$, and very quickly becomes indistinguishable from it. Since the difference $f_{\Delta}(x)=f(x)-c x-c$ looks much like an exponentially damped harmonic function, a graph of $x^{\alpha x} f_{\Delta}(x)$ is displayed in Figure 1c, with the constant $\alpha$ chosen to blow up the shape by approximately the right amount. To further examine the sinusoidal behavior, let \#S designate the cardinality of the set $S$. Then define the function

$$
z(y)=\#\left\{x: f_{\Delta}(x)=0 \text { for } x \leq y\right\} \text { for all } f_{\Delta}(y)=0,
$$

i.e., whenever $y$ is a zero of $f_{\Delta}$ the function $z(y)$ is the number of previous zeros (including the current one). Extend the definition of $z(y)$ to the rest of the points by linear interpolation between the two nearest defined points. If the zeros of $f_{\Delta}$ were equally spaced, $z$ should be a linear function. As the graph of $z-1.175 y$ in Figure $1 \mathrm{f}$ shows, the period of the sine waves is very slowly but steadily decreasing, with the value of nearly 2.35 for values of $y$ up to 200 . Finally, the function $f_{*}(x)=f_{\Delta}(x) / \sin (\pi z(x))$ represents an approximation to the envelope of $f_{\Delta}(x)$, and since it does not have an oscillating sign, one can compute $\ln f_{*}(x) / x \ln x$, displayed in Figure 1e, which indicates that the envelope behaves asymptotically as $x^{\alpha x}$ for some appropriate $\alpha$ near 0.95 .

Perhaps overlooking the oscillatory behavior of $f(x)$, as well as its lack of highorder derivatives, caused Blaisdell and Solomon [4] and Lal and Gillard [10] to put too much faith in the accuracy of their numerical integrations.

5. Dickman's Function. Call this function $v(x)$. It is defined by the differential-difference equation

$$
v(x)=1 \text { for } 0 \leq x \leq 1 \text { and } x v^{\prime}(x)=-v(x-1) \text { for } x>1 .
$$

Dickman [6] gave a heuristic argument for the role of $v(x)$ in analytic number theory. Let $n$ be a large integer and let $p$ be the largest prime factor of an integer $m$ chosen at random from 1 to $n$. Then

$$
\lim _{n \rightarrow \infty} \operatorname{Pr}\left(p \leq n^{1 / x}\right)=v(x) .
$$

For example, if $n$ is an integer near $10^{400}$ and $p$ the largest prime factor of an integer chosen at random from 1 to $n$, then $\operatorname{Pr}\left(p \leq 10^{200}\right) \approx v(2), \operatorname{Pr}\left(p \leq 10^{100}\right) \approx v(4)$ and $\operatorname{Pr}\left(p \leq 10^{10}\right) \approx v(40)$.

The function $v(x)$ is useful in assessing the speed of algorithms for factoring large integers. It was also used by Davenport and Erdös [5] to provide the frequency of residues of a large prime which are $k$ th powers of other residues. For that, the solution of $v(x)=1 / k$ is required. (Should anyone want them, we have solutions 
for $k=2,3, \ldots, 2000$, which may be used to assess the accuracy of the asymptotic results proved in [5].)

Dickman's heuristic result has been made rigorous and extended in numerous papers, for example, Ramaswami [14], Norton [16], Davenport and Erdős [5]. Knuth $[9$, pp. 367-368] gives a nice discussion, using the function $F(t)=v(1 / t)$. Numerical evaluation of $v(x)$ was treated in papers by Bellman and Kotkin [1], van de Lune and Wattel [12] and Lal and Gillard [11].

Since $v(x)$ is convex and rapidly goes to zero, numerical integration should be more effective for it than for the oscillatory solutions to Renyi's or Buchstab's equations. van de Lune and Wattel solved it with great accuracy, as did Lal and Gillard. As mentioned previously, Bellman and Kotkin's numerical solution of Dickman's equation gave unsatisfactory results: they gave, for example, $v(10), v(15), v(20)$ as $0.458,0.202,0.149$, all times $10^{-8}$; the true values are $0.27701 \times 10^{-10}, 0.75899 \times 10^{-18}, 0.24617 \times 10^{-28}$.

We may evaluate $v(x)$ quite easily and very accurately by the series method described above. It is readily verified that, with $v(t)=1$ for $0 \leq t \leq 1$,

$$
v(n+1+t)=v(n+1)-\int_{0}^{t} \frac{v(n+y)}{n+1+y} d y
$$

satisfies condition (8) defining $v$. To get series expansions about the midpoints of intervals, write

$$
v\left(n+1+\frac{1}{2}+\frac{1}{2} z\right)=v(n+1)-\int_{-1}^{z} \frac{v\left(n+\frac{1}{2}+\frac{1}{2} y\right)}{2 n+3+y} d y .
$$

Differentiating this relation and equating powers of $z$ provides simple expressions for $b_{1}, b_{2}, \ldots$ :

$$
b_{1}=-\frac{a_{0}}{2 n+3} \text { and } b_{i}=-\frac{a_{i-1}+b_{i-1}}{i(2 n+3)} \text { for } i=2,3, \ldots
$$

To get $b_{0}$ we express the integrand in (9) as a power series in $y$, getting

$$
v\left(n+1+\frac{1}{2}+\frac{1}{2} z\right)=v(n+1)-\int_{-1}^{z}\left(c_{0}+c_{1} y+c_{2} y^{2}+\ldots\right) d y
$$

with $c_{0}=a_{0} /(2 n+3)$ and $c_{i}=\left(a_{i}-c_{i-1}\right) /(2 n+3)$ for $i=1,2,3, \ldots$. Then

$$
b_{0}=\frac{1}{2 n+3}\left[a_{0}\left(1+c_{0} / 1\right)+a_{1}\left(1-c_{1} / 2\right)+a_{2}\left(1+c_{2} / 3\right)+\ldots\right] .
$$

The sum for $b_{0}$ may be computed without declaring an array for the $c^{\prime} s$, since they may be built up recursively and are not needed other than for evaluating $b_{0}$.

As with Renyi's function, the coefficients for successive intervals of Dickman's function were built up recursively, with care taken to provide over a thousand digits of accuracy. Dickman's function $v(x)$ is displayed in Fig. 1b. The function $\ln v(x) / x \ln x$ is shown in Fig. 1e, and reveals that $v(x)$ behaves asymptotically as $x^{\alpha x}$.

6. The $U_{1}+U_{1} U_{2}+U_{1} U_{2} U_{3}+\cdots$ Function. Dickman's function arises in a completely different setting, with different initial conditions: as the density of the random variable $X$, defined by

$$
X=U_{1}+U_{1} U_{2}+U_{1} U_{2} U_{3}+\cdots,
$$


where $U_{1}, U_{2}, U_{3}, \ldots$ are independent random variables, each uniformly distributed on $(0,1)$. Let $g(x)$ be the density of $X$. Since $U_{1}(1+X)$ has the same distribution as $X$, we may write, when $x>1$,

$$
\begin{aligned}
\operatorname{Pr}[X<x] & =\int_{0}^{\infty} \operatorname{Pr}\left[U_{1}(1+X)<x \mid X=t\right] g(t) d t \\
& =\int_{0}^{x-1} g(t) d t+\int_{x-1}^{\infty} \frac{x}{1+t} g(t) d t
\end{aligned}
$$

Differentiation with respect to $x$ gives the density function for $X$ when $x>1$ :

$$
g(x)=\int_{x-1}^{\infty} \frac{g(t)}{1+t} d t
$$

It follows that $g(x)$ satisfies Dickman's differential-difference equation, $x g^{\prime}(x)=$ $-g(x-1)$, but with initial conditions yet to be determined. To establish the initial conditions, note that when $0 \leq x \leq 1$, Eq. (11) reduces to

$$
g(x)=\frac{d}{d x}\left(x \int_{0}^{\infty} \frac{g(t)}{1+t} d t\right)=\text { constant }
$$

To find that constant, $g(0)$, we find the Laplace transform of $g(x)$, say $\phi(s)$, and use the relation $g(0)=\lim _{s \rightarrow \infty} s \phi(s)$. From the relation $x g^{\prime}(x)=-g(x-1)$ it is elementary to derive a differential equation for $\phi(s)$, the Laplace transform of $g(x)$ :

$$
s \phi^{\prime}(s)=\left(e^{-s}-1\right) \phi(s) \text { and thus } \phi(s)=\exp \left(-\int_{0}^{t} \frac{1-e^{-y}}{y} d y\right) .
$$

(The additive constant in solving the differential equation is zero, since $\phi(0)=$ $\int_{0}^{\infty} g(x) d x=1$.) The exponent of $e$ in $\phi(s)$ may be integrated by parts to yield $\left(1-e^{-s}\right) \ln s-\int_{0}^{t} e^{-y} \ln y d y$, so that

$$
g(0)=\lim _{s \rightarrow \infty} s \phi(s)=\exp \left(-\int_{0}^{\infty} e^{-y} \ln y d y\right)=e^{-\gamma}
$$

with $\gamma=\int_{0}^{\infty} e^{-y} \ln y d y$, Euler's constant in one of its many manifestations.

Since $g(x)$ and Dickman's function $v(x)$ differ only in that their initial values are, respectively, $e^{-\gamma}$ and 1 , values of $g(x)$ may be obtained by $g(x)=e^{-\gamma} v(x)$.

7. Buchstab's Function. The function defined by

$$
w(x)=1 / x \text { for } 1 \leq x \leq 2 \text { and }[x w(x)]^{\prime}=w(x-1) \text { for } 2 \leq x
$$

was shown by Buchstab [3] and Selberg [17] to be the limiting form for the number of uncancelled elements in the sieve of Eratosthenes in particular cases. These results were extended by De Bruijn [2], who showed, among other things, that $\lim _{x \rightarrow \infty} w(x)=e^{-\gamma}$.

The series method is readily applied to $w(x)$ and gives very accurate values over successive intervals 2 to 3,3 to 4 , etc. We have, for $n=0,1,2, \ldots$ and $0 \leq t \leq 1$,

$$
(n+1+t) w(n+1+t)=(n+1) w(n+1)+\int_{0}^{t} w(n+y) d y
$$


and thus

$$
\begin{aligned}
(2 n+3+z) w\left(n+1+\frac{1}{2}+\frac{1}{2} z\right) & \\
& =(2 n+2) w(n+1)+\int_{-1}^{z} w\left(n+\frac{1}{2}+\frac{1}{2} u\right) d u
\end{aligned}
$$

For given $n$, if

$$
w\left(n+\frac{1}{2}+\frac{1}{2} z\right)=a_{0}+a_{1} z+a_{2} z^{2}+\cdots
$$

and we want $w$ for the next interval:

$$
w\left(n+1+\frac{1}{2}+\frac{1}{2} z\right)=b_{0}+b_{1} z+b_{2} z^{2}+\cdots,
$$

then we may substitute the $a$ series for the integrand in (13), then integrate and equate coefficients of like powers of $z$ to obtain

$$
\begin{gathered}
b_{0}=\frac{1}{2 n+3}\left[(2 n+2+1) a_{0}+(2 n+2-1 / 2) a_{1}+(2 n+2+1 / 3) a_{2}+\cdots\right], \\
b_{i}=\left(a_{i-1} / i-b_{i-1}\right) /(2 n+3) \quad \text { for } i=1,2,3, \ldots
\end{gathered}
$$

Developing series for successive intervals in this way, we have used our extended precision routines on a PC AT to evaluate Buchstab's function out to $x=500$. That function, $w(x)$, is displayed in Figure 1b. Like Renyi's function $f(x)$, it oscillates as it approaches its limiting value, $e^{-\gamma}$, and the difference $w_{\Delta}(x)=w(x)-e^{-\gamma}$ looks like a damped harmonic function. The function $x^{\alpha x} w_{\Delta}(x)$, with $\alpha$ selected to make the scale reasonable, is shown in Figure 1d. The zero-counting function $z(y)$ and the envelope $w_{*}(x)$ are defined as they were for Renyi's function. Figure If is $z(y)-1.175 y$ which shows that the zeros are almost evenly spaced with the spaces diminishing slowly as $y$ increases. (We do not yet know whether the limiting spacing is zero or some positive constant.) A graph of $\ln w_{*}(x) / x \ln x$ in Figure 1e shows that the envelope also behaves asymptotically as $x^{\alpha x}$, with the limiting value for alpha near 0.82 .

We found the published results of Lal and Gillard [11] to be accurate to the specified 25 places. As a check on our method of solving the equation, we compared the value at $x=500$ with the known asymptotic value, $e^{-\gamma}$ and found agreement to at least 1000 digits. (Since $\gamma$ is known to several thousand digits - for example, Sweeney [18]-it is relatively easy to find $e^{-\gamma}$ to 1000 digits.)

8. Addendum. If $h(t)=\exp \left(-2 \int_{0}^{t} \frac{1-e^{-x}}{x} d x\right)$, then Renyi's constant is $c=$ $\int_{0}^{\infty} h(t) d t$. We evaluated $c$ with great accuracy above, as the limiting slope of the solution to Renyi's differential-difference equation. To confirm that accuracy, and to support our contention that published values of that constant, [4] and [10], are far off the mark, we now find $c$ to 100 places by evaluating $\int_{0}^{\infty} h(t) d t$ directly.

The method is as follows:

$$
c=\int_{0}^{1} h(t) d t+\int_{1}^{2} h(t) d t+\cdots+\int_{230}^{231} h(t) d t+\int_{231}^{\infty} h(t) d t
$$


(The choice 231 comes from $e^{-231}<10^{-100}$, used in bounding the tail integral.) Each of the integrals, $\int_{k}^{k+1} h(t) d t, k=0,1,2, \ldots, 230$ may be evaluated with great accuracy by expanding $h(t)$ in a Taylor series about $t=k$, leading to

$$
\int_{k}^{k+1} h(t) d t=\int_{0}^{1} h(k+x) d x=h(k)+h^{\prime}(k) / 2 !+h^{\prime \prime}(k) / 3 !+\cdots .
$$

For a fixed $k$, define the derivatives $h_{0}=h(k)$ and $h_{n}=\left.h^{(n)}(t)\right|_{t=k}$. The $h_{n}$ may be developed recursively:

$$
h_{n+1}=-2 \sum_{j=0}^{n}\left(\begin{array}{c}
n \\
j
\end{array}\right) g_{j} h_{n-j}
$$

where $g(t)=\left(1-e^{-t}\right) / t$ and $g_{n}=\left.g^{(n)}(t)\right|_{t=k}$ satisfies $g_{0}=1$ and $g_{n}=\left(-n g_{n-1}-\right.$ $\left.(-1)^{n} e^{-k}\right)$.

These recursions allow, for each $k$, successive derivatives of $h$ to be built up as exact, rational multiples of $h(k)$. This provides an alternating series for $\int_{k}^{k+1} h(t) d t$ with error that may be controlled by the error in evaluating $h(k)$ (essentially the exponential integral function, about which much is known), and the error from neglecting the tail of an alternating series.

To evaluate $h(k)$ with great accuracy, we write

$$
h(t)=\exp \left(-2 \int_{0}^{t} \frac{1-e^{-x}}{x} d x\right)=e^{-2 \gamma-2 \ln (t)+2 \operatorname{Ei}(-t)},
$$

and then use well-known methods for evaluating $\operatorname{Ei}(-k)=E_{1}(k)$ at the points $k=0,1,2, \ldots, 230$. Our rational arithmetic procedures provided each of the $\operatorname{Ei}(-k)$ values with error less than $10^{-110}$. Then adding $\int_{0}^{1}+\int_{1}^{2}+\cdots+\int_{230}^{231}$ provides $\int_{0}^{231} h(t) d t$ with an error less than $10^{-104}$.

Finally, we need the tail integral, $\int_{231}^{\infty} h(t) d t$, with an error less than $10^{-101}$. We combine Eqs. 8.212(1) and 8.212(3) of [8, p. 925], to write

$$
\int_{0}^{t} \frac{1-e^{-x}}{x} d x=\gamma+\ln t+e^{-t} / t-r(t)
$$

where

$$
r(t)=e^{-t} \int_{0}^{\infty} \frac{e^{-x}}{(t+x)^{2}} d x .
$$

Note that $r(t)<e^{-t} / t^{2}$ for $t>0$, and that for $t>231$,

$$
1<e^{2 r(t)}<e^{2 e^{-231} / 231^{2}}<1+1.8 \times 10^{-105} .
$$

We may write the tail integral in the form

$$
\int_{231}^{\infty} h(t) d t=e^{-2 \gamma} \int_{231}^{\infty} t^{-2} e^{-2 e^{-t} / t+2 r(t)} d t .
$$

With $V=e^{-2 \gamma} \int_{231}^{\infty} t^{-2} e^{-2 e^{-t} / t} d t$, relation (14) provides the bounds

$$
V<\int_{231}^{\infty} h(t) d t<V+1.8 V \times 10^{-105} .
$$



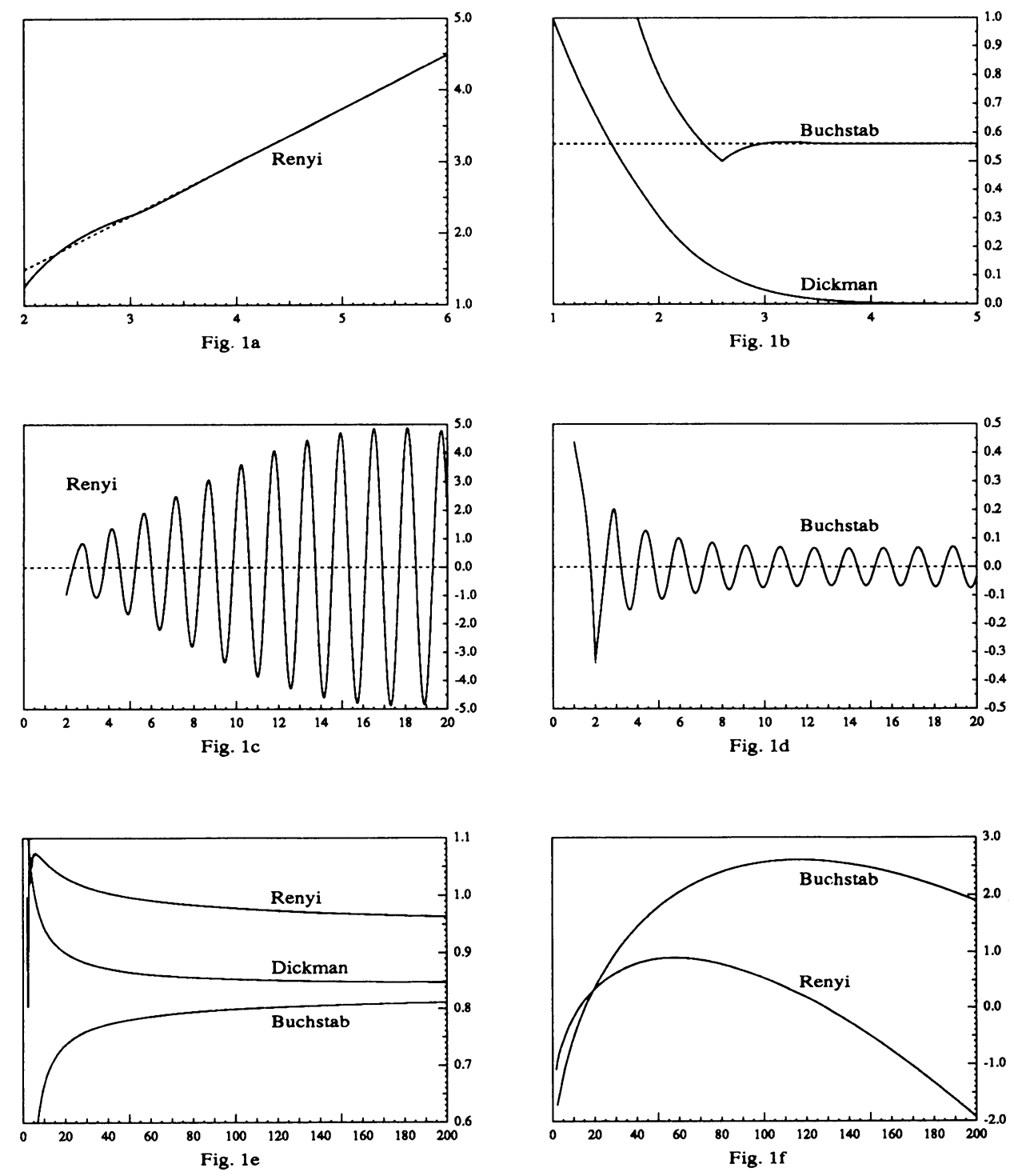

FIGURE 1

Graphs of the Renyi, Dickman, and Buchstab functions

$(1 \mathrm{a}, 1 \mathrm{~b})$, with related functions $(1 \mathrm{c}, 1 \mathrm{~d}, 1 \mathrm{e}, 1 \mathrm{f})$.

Details are in the text.

Thus if we find $V$ to within $10^{-101}$ we will have the tail integral to the required 100-place accuracy.

To evaluate $V$, we expand $e^{-2 e^{-t} / t}$ in a series to get

$$
V=e^{-2 \gamma} \int_{231}^{\infty}\left[\frac{1}{t^{2}}-\frac{2 e^{-t}}{t^{3}}+\frac{4 e^{-2 t}}{2 t^{4}}-\cdots\right] d t
$$

Term-by-term integration yields an alternating series of decreasing magnitudes, so that the error in using only the first term, $e^{-2 \gamma} / 231$, is less than the second term, which is bounded by $2.44 \times 10^{-108}$. 
Thus we have a direct representation of Renyi's constant:

$$
c=\int_{0}^{231} h(t) d t+\frac{e^{-2 \gamma}}{231}+\varepsilon,
$$

with $|\varepsilon|<10^{-100}$. The value obtained by this method agrees with the 100 -place value of $c$ provided by our numerical solution of Renyi's equation, displayed in Section 4.

Supercomputer Computations Research Institute and Department of Statistics The Florida State University Tallahassee, Florida 32306

E-mail: geo@stat.stat.fsu.edu arif@stat.stat.fsu.edu

Department of Computer Science

Western Oregon State College

Monmouth, Oregon 97361

1. R. BeLLMAN \& B. KOTKIN, "On the numerical solution of a differential-difference equation arising in analytic number theory," Math. Comp., v. 16, 1962, pp. 473-475.

2. N. G. DE BRUIJN, "On the number of uncancelled elements in the sieve of Eratosthenes," Nederl. Akad. Wetensch. Proc., v. 53, 1950, pp. 803-812; Indag. Math., v. 12, 1950, pp. 247-256.

3. A. BuchstaB, "Asymptotic estimates of a general number-theoretic function," Mat. Sb. (N.S.), v. 44, 1937, pp. 1239-1246. (In Russian)

4. B. E. BlAisDell \& H. SOlOMON, "On random sequential packing in the plane and a conjecture of Palesti," J. Appl. Probab., v. 7, 1970, pp. 667-698.

5. H. DAVENPORT \& P. ERDős, "The distribution of quadratic and higher residues," Publ. Math. Debrecen, v. 2, 1951-1952, pp. 252-265.

6. K. DICKMAN, "On the frequency of numbers containing prime factors of a certain relative magnitude," Ark. Mat. Astronom. Fys., v. 22A, 10, 1930, pp. 1-14.

7. A. DVOREtSKy \& H. ROBbins, "On the 'parking' problem," Publ. Math. Inst. Hungar. Acad. Sci., v. 9, 1964, pp. 209-226.

8. I. S. GRADShteyn \& I. M. RYZhiK, Tables of Integrals, Series, and Products, Academic Press, New York and London, 1965.

9. D. E. KNUth, The Art of Computer Programming, Volume 2: Seminumerical Algorithms, Addison-Wesley, Reading, Mass., 1981.

10. M. LAL \& P. GILLARD, "Evaluation of a constant associated with a parking problem," Math. Comp., v. 28, 1974, pp. 561-564.

11. M. LAL \& P. GILlaRD, Numerical Solution of Two Differential-Difference Equations of Analytic Theory of Numbers, Lecture Notes in Math., vol. 109, Springer-Verlag, Berlin and New York, 1969, pp. 179-187.

12. J. VAN DE LUNE \& E. WATTEL, "On the numerical solution of a differential-difference equation arising in analytic number theory," Math. Comp., v. 23, 1969, pp. 417-421.

13. David Manion, "Random space-filling in one dimension," Publ. Math. Inst. Hungar. Acad. Sci., v. 9, 1964, pp. 143-153.

14. V. RAMASWAMI, "On the number of positive integers less than $x$ and free of prime factors greater than $x^{e}$," Bull. Amer. Math. Soc., v. 55, 1949, pp. 1122-1127.

15. AlfRED RENYI, "On a one-dimensional problem concerning random space filling," Publ. Math. Inst. Hungar. Acad. Sci., v. 3, 1958, pp. 109-127. (In Hungarian)

16. K. K. NORTON, Numbers with Small Prime Factors and Least $k$-th Power Non-Residues, Mem. Amer. Math. Soc., vol. 106, Amer. Math. Soc., Providence, R.I., 1971, pp. 9-27.

17. S. SELBERG, "The number of cancelled elements in the sieve of Eratosthenes," Norsk. Mat. Tidsskr., v. 26, 1944, pp. 79-84. (In Norwegian)

18. D. W. SWEENEY, "On the computation of Euler's constant," Math. Comp., v. 17, 1963, pp. 170-178. 